\title{
What is the minimal clinically important difference for helium-3 magnetic resonance imaging ventilation defects?
}

\author{
To the Editor:
}

Pulmonary magnetic resonance imaging (MRI) using inhaled polarised gases provides a way to directly visualise and sensitively measure lung ventilation abnormalities or ventilation defects [1]; the burden in individual patients may be directly quantified as the percent ventilation volume [2], ventilation defect volume (VDV) [3] or ventilation defect percent (VDP) [4], which is VDV normalised to the total lung volume. In patients with asthma, MRI ventilation defects worsen during methacholine [5] and exercise challenge $[5,6]$, and respond to bronchodilation [5, 6]. However, it is still unknown if quantitative changes in MRI ventilation abnormalities directly reflect changes in patient-related outcomes like symptoms; this is important when considering MRI for clinical and research studies in asthma patients, which require an understanding of the minimal clinically important difference (MCID).

First described in 1989 [7], the MCID reflects the smallest measurement difference that patients perceive as beneficial. MCID estimations typically involve patient perception but up to nine methods have been reported [8] and no standard for calculating MCID has been established. For example, changes in clinical parameters provide the foundation for the so-called anchor-based MCID approach [9], in which patientor clinician-reported metrics serve as "anchors". Conversely, distribution-based or data-driven approaches reflect instrument error and precision, including the standard error of measurement (SEM) [10], which has been validated as a proxy for the MCID.

Here, we estimated the MCID of MRI VDV and VDP using distribution- and anchor-based approaches. We used both approaches because MRI VDV and VDP measurement precision are heavily dependent on the algorithm used and the reproducibility of the quantification. First, we used the SEM to estimate the distribution-based MCID for VDV based on algorithm precision previously published [3]. As previously described [3], pulmonary MRI VDV is quantified using a semiautomated algorithm in units of millilitres while VDP is measured as a percentage of the thoracic cavity volume in units of percentage. Based on five repeated segmentation rounds in 15 subjects, the SEM for VDP was calculated as the square root of the repeated measures intraobserver VDP variance and was $40 \mathrm{~mL}$ [3]. We also consider the smallest detectable difference (SDD), which generates confidence about measurement uncertainty. The previously calculated SDD for VDV was $110 \mathrm{~mL}$ [3] and because this is larger than the SEM, it is possible that an observed change less than the SEM would be due to measurement error. In contrast, if the SDD is smaller than the MCID, it is possible to distinguish a clinically important change with adequate measurement precision. To be certain that a clinically important change is not due to measurement error, we propose to conservatively use the MCID of $110 \mathrm{~mL}$, which reflects measurement precision, instead of $40 \mathrm{~mL}$, which was the measured SEM.

For the anchor-based method, we used the patient-reported and validated Asthma Control Questionnaire (ACQ) score [11] as the anchor, and the significant relationship between ACQ score and MRI VDP previously published in 18 asthmatic patients [12]. In these asthmatics, the relationship between ACQ score and VDP was determined by the equation of their linear relationship as VDP=7.5 $\times$ ACQ-5.0 [12]. The MCID for ACQ score was previously determined to be 0.5 [11] and therefore, based on the linear

@ERSpublications

In asthmatics, the estimated MCID for ${ }^{3} \mathrm{He}$ MRI ventilation defect volume is $110 \mathrm{~mL}$ and that for ventilation defect percentage is $2 \%$, which are similar to $\mathrm{FEV}_{1}$, suggesting that these biomarkers are suitable for use in clinical trials http://ow.ly/yQ8K30jAFnX

Cite this article as: Eddy RL, Svenningsen S, McCormack DG, et al. What is the minimal clinically important difference for helium-3 magnetic resonance imaging ventilation defects? Eur Respir J 2018; 51: 1800324 [https://doi.org/10.1183/13993003.00324-2018]. 
relationship, a 0.5 change in ACQ would result in a 4\% VDP difference. Therefore, using ACQ score as an anchor, the VDP MCID is $4 \%$.

While ACQ score and exacerbations may be used in asthma clinical trials, the most commonly used objective end-point is the forced expiratory volume in $1 \mathrm{~s}$ (FEV1); the MCID for FEV 1 is often described as a range which for asthmatics is $100-200 \mathrm{~mL}$ [13]. In contrast with FEV1, which is dominated by the large airways [14], MRI is sensitive to all airways and the MCID is $110 \mathrm{~mL}$ for VDV (distribution-based) and $4 \%$ for VDP (anchor-based). The 4\% VDP MCID can be translated to a VDV of $200 \mathrm{~mL}$ based on the mean thoracic cavity volume segmented from MRI, which was reported to be $5.0 \mathrm{~L}$ [3]. In a similar manner, the VDV MCID of $110 \mathrm{~mL}$ is equivalent to $\sim 2 \%$. Thus, we report a range of $110-200 \mathrm{~mL}$ for VDV and 2-4\% for VDP as MCID ranges. To illustrate the quantitative meaning of the MCID of VDP in individual patients, figure 1 shows MRI for three patients with asthma with visually and quantitatively improved ventilation following bronchodilation (increasing VDP improvement shown from left to right). For subject S1, there was a change in VDV/VDP equivalent to the distribution-based MCID or SDD. For subjects S2 and S3, there were post-bronchodilator changes in VDV/VDP that were similar in magnitude to the anchor-based MCID estimate. Notably, S1 and S3 showed clinically significant post-bronchodilator FEV1 changes $(\geqslant 200 \mathrm{~mL}$ and $\geqslant 12 \%)$, while S2 did not. The sensitivity of MRI to post-bronchodilator changes highlights a unique opportunity for pulmonary MRI to help explain subjective or patient-perceived improvements (i.e. ACQ or quality of life improvements) that are not reflected by FEV1.

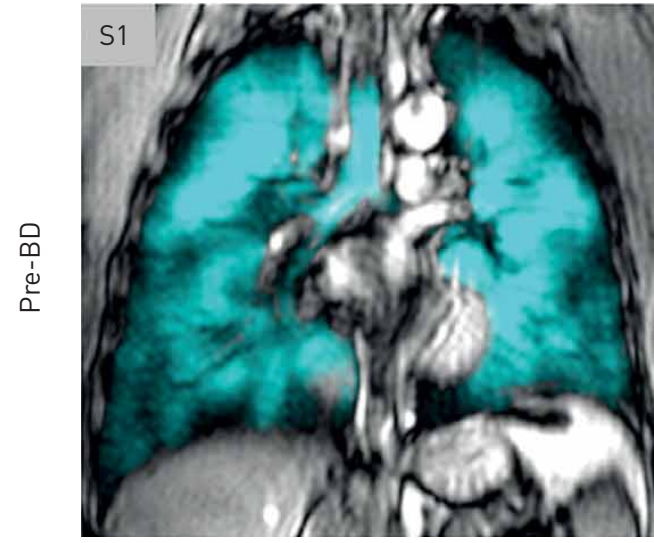

FEV1 $2.06 \mathrm{~L}$ VDV $390 \mathrm{~mL}$ VDP $8 \%$

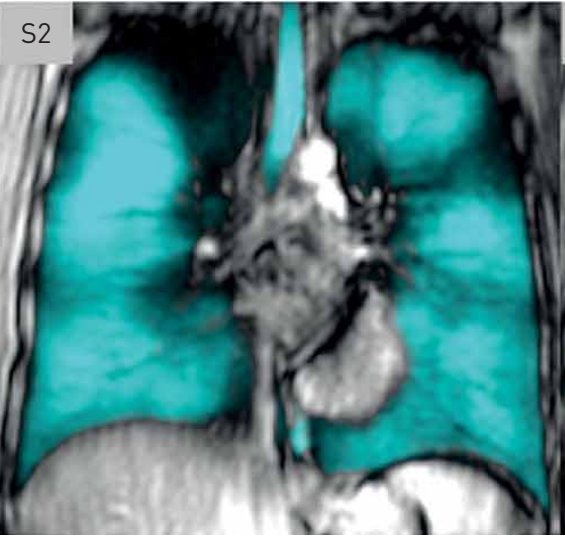

FEV1 $2.37 \mathrm{~L}$ VDV $240 \mathrm{~mL}$ VDP $6 \%$

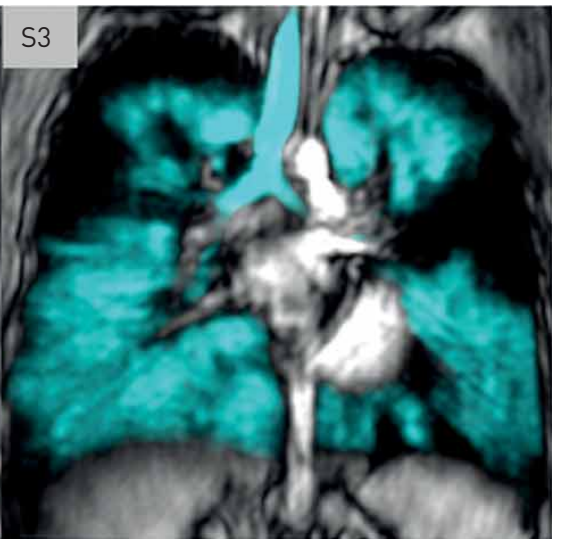

FEV1 $0.94 \mathrm{~L}$ VDV $580 \mathrm{~mL}$ VDP $13 \%$

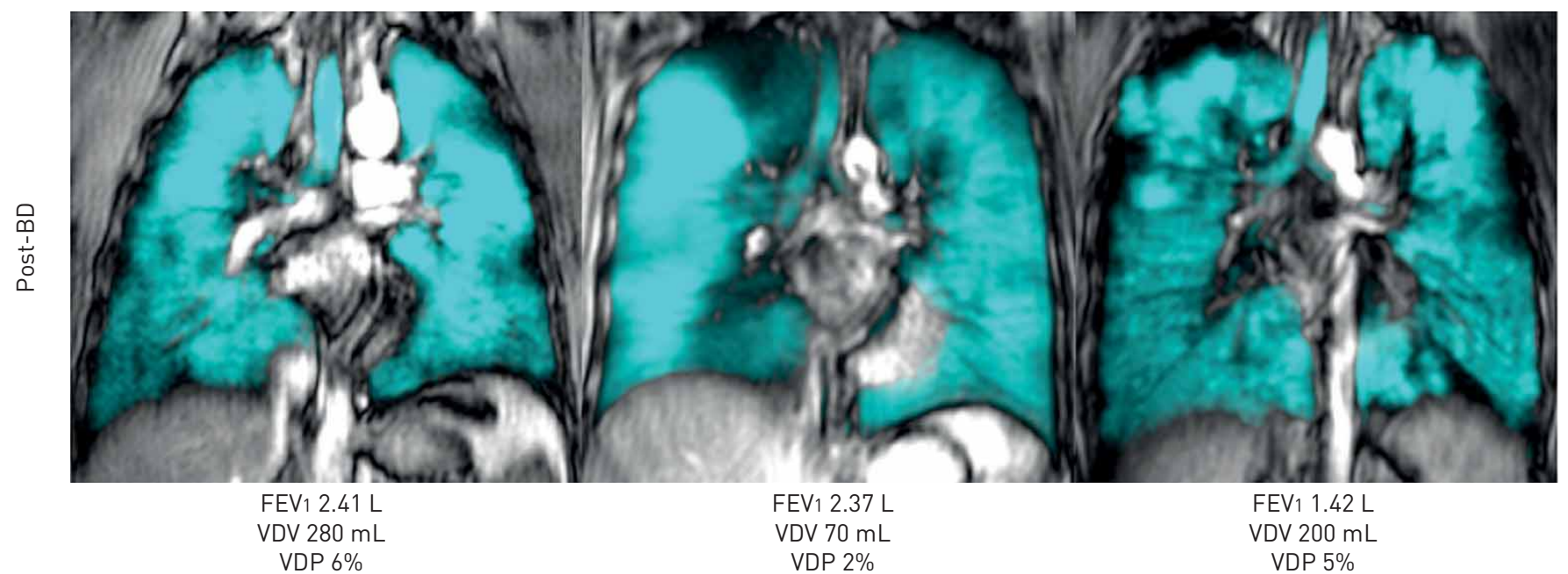

FIGURE 1 Change in asthmatic magnetic resonance imaging (MRI) ventilation after bronchodilator (BD) for three representative subjects. Three asthmatic subjects exhibited visual changes in MRI ventilation post-BD. A 45-year-old male (S1) underwent an improvement in ventilation equal to the smallest detectable difference and distribution-based minimal clinically important difference (MCID), while a 28-year-old female (S2) and a 31-year-old female (S3) underwent improvements at least as large as the anchor-based MCID. Notably, S1 and S3 also exhibited clinically significant changes in forced expiratory volume in $1 \mathrm{~s}$ (FEV1) ( $\geqslant 200 \mathrm{~mL}$ and $\geqslant 12 \%)$ but $\mathrm{S} 2$ did not. VDV: ventilation defect volume; VDP: ventilation defect percent. 
The number of experts using hyperpolarised gas MRI in asthma clinical trials is still very small so it is important to consider the MRI VDP MCID in the context of the MCID of other established asthma biomarkers. The MCID we calculated for MRI VDP is similar to the MCID for FEV1 in asthma at 110$200 \mathrm{~mL}$. Moreover, we have used the ACQ MCID of 0.5 [11] to determine the upper limit of this range at $200 \mathrm{~mL}$ and therefore, these are already intrinsically related. The MCID for the Asthma Quality of Life Questionnaire (AQLQ) is also 0.5 [15], and though the relationship between VDP and AQLQ has not been directly established, we previously showed that VDP is significantly worse in patients with lower quality of life (AQLQ <5) [12]. MRI VDP directly detects early changes in clinically important pathologies with high reproducibility [16]. Taken together, this means that MRI has both the sensitivity and precision needed for clinical studies, although the complexity and cost of the acquisition of these measurements compared to other tests is still a limitation.

It is important to consider the helium-3 MRI results in the context of future development of xenon-129 MRI, which is much less costly to acquire. In this regard, we previously directly compared ${ }^{3} \mathrm{He}$ and ${ }^{129} \mathrm{Xe}$ MRI, and showed that ${ }^{129} \mathrm{Xe}$ VDP was greater than ${ }^{3} \mathrm{He}$ VDP in asthmatics [17]; this suggested that there was enhanced sensitivity to airway abnormalities using ${ }^{129} \mathrm{Xe}$ gas, which we speculated was due to the viscosity and diffusivity of the gas, so that ${ }^{129} \mathrm{Xe}$ VDP was systematically larger than ${ }^{3} \mathrm{He}$ VDP in asthmatics. Based on this important information, we speculate that the slope of the linear relationship between ACQ and ${ }^{129} \mathrm{Xe}$ VDP, and thus the MCID, would be similar to ${ }^{3} \mathrm{He}$ MRI VDP, but these calculations still need to be undertaken in a prospective ${ }^{129} \mathrm{Xe}$ MRI study. It is also important to note that, though there is no standard for calculating MCID values, the anchor-based estimation we generated here was based on cross-sectional data and did not reflect within-subject variability or response to therapy. Considering the original definition of MCID [7], "within-subject" differences in response to therapy will be important to investigate in prospectively designed clinical trials.

In summary, pulmonary MRI biomarkers of ventilation have already provided some intriguing results in patients with asthma, but to our knowledge, MRI biomarkers have not been used in large-scale clinical trials of potential new therapies. Other considerations aside (i.e. technological and financial), this lack of uptake may reflect the lack of a deep understanding of the relationship between MRI biomarkers with how patients perceive symptoms. We provide calculations of MCID for ${ }^{3} \mathrm{He}$ MRI VDV and VDP to support the use of MRI in the research and development of novel therapies, as well as therapy decisions or $n=1$ trials, towards more precise decision making in individual patients.

Rachel L. Eddy ${ }^{1,2}$, Sarah Svenningsen ${ }^{1,2,3}$, David G. McCormack ${ }^{4}$ and Grace Parraga ${ }^{1,2,4}$

${ }^{1}$ Robarts Research Institute, Western University, London, ON, Canada. ${ }^{2}$ Dept of Medical Biophysics, Western University, London, ON, Canada. ${ }^{3}$ Division of Respirology, Dept of Medicine, McMaster University, Hamilton, ON, Canada. ${ }^{4}$ Division of Respirology, Dept of Medicine, Western University, London, ON, Canada.

Correspondence: Grace Parraga, Robarts Research Institute, 1151 Richmond St N, London N6A 5B7, Canada. E-mail: gparraga@robarts.ca

Received: Feb 142018 | Accepted after revision: April 032018

Conflict of interest: None declared.

\section{References}

1 Kauczor H-U, Ebert M, Kreitner K-F, et al. Imaging of the lungs using ${ }^{3} \mathrm{He}$ MRI: preliminary clinical experience in 18 patients with and without lung disease. J Magn Reson Imaging 1997; 7: 538-543.

2 Woodhouse N, Wild JM, Paley MN, et al. Combined helium-3/proton magnetic resonance imaging measurement of ventilated lung volumes in smokers compared to never-smokers. J Magn Reson Imaging 2005; 21: 365-369.

3 Kirby M, Heydarian M, Svenningsen S, et al. Hyperpolarized ${ }^{3} \mathrm{He}$ magnetic resonance functional imaging semiautomated segmentation. Acad Radiol 2012; 19: 141-152.

4 Fain SB, Gonzalez-Fernandez G, Peterson ET, et al. Evaluation of structure-function relationships in asthma using multidetector CT and hyperpolarized He-3 MRI. Acad Radiol 2008; 15: 753-762.

5 Samee S, Altes T, Powers P, et al. Imaging the lungs in asthmatic patients by using hyperpolarized helium-3 magnetic resonance: assessment of response to methacholine and exercise challenge. J Allergy Clin Immunol 2003; 111: 1205-1211.

6 Kruger SJ, Niles DJ, Dardzinski B, et al. Hyperpolarized helium-3 MRI of exercise-induced bronchoconstriction during challenge and therapy. J Magn Reson Imaging 2014; 39: 1230-1237.

7 Jaeschke R, Singer J, Guyatt GH. Measurement of health status. Ascertaining the minimal clinically important difference. Control Clin Trials 1989; 10: 407-415.

8 Wells G, Beaton D, Shea B, et al. Minimal clinically important differences: review of methods. J Rheumatol 2001; 28: 406-412.

9 Copay AG, Subach BR, Glassman SD, et al. Understanding the minimum clinically important difference: a review of concepts and methods. Spine J 2007; 7: 541-546.

10 Wyrwich KW, Nienaber NA, Tierney WM, et al. Linking clinical relevance and statistical significance in evaluating intra-individual changes in health-related quality of life. Med Care 1999; 37: 469-478. 
11 Juniper EF, Svensson K, Mork AC, et al. Measurement properties and interpretation of three shortened versions of the asthma control questionnaire. Respir Med 2005; 99: 553-558.

12 Svenningsen S, Nair P, Guo F, et al. Is ventilation heterogeneity related to asthma control? Eur Respir J 2016; 48: 370-379.

13 Jones PW, Beeh KM, Chapman KR, et al. Minimal clinically important differences in pharmacological trials. Am J Respir Crit Care Med 2014; 189: 250-255.

14 Macklem PT, Mead J. Resistance of central and peripheral airways measured by a retrograde catheter. J Appl Physiol 1967; 22: 395-401.

15 Juniper EF, Guyatt GH, Willan A, et al. Determining a minimal important change in a disease-specific quality of life questionnaire. J Clin Epidemiol 1994; 47: 81-87.

16 de Lange EE, Altes TA, Patrie JT, et al Changes in regional airflow obstruction over time in the lungs of patients with asthma: evaluation with ${ }^{3} \mathrm{He}$ MR imaging. Radiology 2009; 250: 567-575.

17 Svenningsen S, Kirby M, Starr D, et al Hyperpolarized ${ }^{3} \mathrm{He}$ and ${ }^{129}$ Xe MRI: differences in asthma before bronchodilation. J Magn Reson Imaging 2013; 38: 1521-1530. 